\title{
EDITORIAL
}

\section{Markov Processes and Related Topics}

\section{Mu-Fa CHEN}

School of Mathematical Sciences, Beijing Normal University, Laboratory of Mathematics and Complex Systems, Ministry of Education, Beijing 100875, China

(C) Higher Education Press and Springer-Verlag Berlin Heidelberg 2014

The "Workshop on Markov Processes and Related Topics" were held nine times since 2002, organized mainly by the Probability Group in the Research Center for Stochastics, Beijing Normal University. The tenth will be held in the coming August, 2014. This workshop happens almost in each year except 2003 (cancelled because of SARS), and 2009 (cancelled because too many related conferences were held in that year). In 2005, the 3rd workshop is merged into the Joint Meeting of CSPS/IMS. The research group was mainly supported by the National Natural Science Foundation of China (NSFC) in the period of 2002-2010, and by the Ministry of Education (985 project) during 2012-2014. Among the ten workshops, five of them were or will be co-organized with some universities out of Beijing.

The 4th (2006), Central South University

The 6th (2008), Anhui Normal University

The 8th (2012), Fujian Normal University

The 9th (2013), Southwest Jiaotong University

The 10th (2014), Xidian University

It is greatly appreciated that these universities kindly provide partially financial supports for the workshops. Many probabilists from different parts of world have participated and made great contributions to the workshops. At each time, there are about 80 participants, including both senior professors and young graduate students. Some of those from abroad have participated each of the workshops, this is certainly not easy as one can imaging. Without the great effort and encouragement by the participants, it is hardly believable that the workshop can last for such a long period. To record the workshops, we are compiling a small electronic book including a list of participants, abstracts of the talks, and photos from the workshops of each time. Once ready, we will put the book on our group's homepage:

http://math.bnu.edu.cn/probab/Anniversary/

\footnotetext{
Received March 8, 2014

E-mail: mfchen@bnu.edu.cn
} 
With the support from the editorial board and office of the journal Frontiers of Mathematics in China, we are now able to organize a special issue for the workshops in this journal (and another issue in the next year).

It may be a good place to list the members of our research group not only for their contributions to organizing the workshops but also for their scientific contributions to the group. In 2001, when we applied for the group's research grant from NSFC, the names listed on the application form are the following: $\mathrm{Mu}-\mathrm{Fa}$ Chen, Fengyu Wang, Zenghu Li, Yuhui Zhang, Yonghua Mao, Wenming Hong, and Yingzhe Wang (seven people), with group's adviser Zikun Wang. In the past ten years or more, four new members have joined our group: Mei Zhang (2004), Jinghai Shao (2006), Yutao Ma (2007), and Hui He (2008). In the past 15 years or so, our secretary Wenchuang Zhou has done a great and successful job for the group. Say thanks to all of them. 